\title{
Accesibilidad a ventanas ecocardiográficas transtorácicas intraoperatorias en cirugía abdominal bajo anestesia general
}

\author{
Accessibility to echocardiographic intraoperative \\ transthoracic windows in abdominal surgery under \\ general anesthesia
}

Mauro M. Costantini', Martín Carpinella'

\begin{abstract}
Background and Objetive: There is enough bibliography that supports the use of Transthoracic echocardiography (TTE) and the impact it generates in the field of critical medicine. However, the main limitation in anesthesia is access to windows in patients with unchanged positions and usually in mechanical ventilation. Therefore we decided to conduct an observational study regarding the obtaining of basic echocardiographic windows in patients under general anesthesia. Methods: 50 patiens were enrolled. After ventilator setting, in dorsal decubitus and with the surgical fields placed, 4 windows were explored: subcostal, apical, parasternal and supraesternal. PEEP, tidal volume, BMI, age, sex were recorded. Each window was evaluated with a score: 2 points: optimal, 1 point: partial, 0 point: the window can not be obtained. Results: The subcostal window could not be obtained in any patient (surgical fields). The remaining 3 windows on score of 6 possible points the average was 5.14. The parasternal window obtained a lowest score $(p=0,0026)$. The most frequent cause of impossibility of access to the window was the presence of the lung. There was no difference between subgroups (PEEP > 10 and $\mathrm{BMI}>30$ ) and score obtained, according to Pearson coefficient. Conclusion: The accessibility to the apical and suprasternal windows was close to optimal. The parasternal window had smaller scores but had no relation to the level of PEEP nor the BMI. Limitations: small number of patients and very limited shelter to extrapolate findings.
\end{abstract}

Key words: Accessibility, transthoracic echocardiography, general anesthesia

Anestesiólogos

Hospital Privado de Comunidad. Mar del Plata. Argentina.

Fecha de ingreso: 20 de mayo de 2018

Fecha de aceptación: 04 de julio de 2018

ORCID

https://orcid.org/0000-0002-1321-5962

Correspondencia:

Dr. Mauro Constantini

E-mail: drmaucos@icloud.com 


\section{RESUMEN}

Introducción y Objetivo: Existe suficiente bibliografía que avala el uso de la ecocardiografía transtorácica y el impacto que genera en el campo de la medicina crítica. Sin embargo, la principal limitante es el limitado acceso a las ventanas en pacientes en posiciones inmodificables y habitualmente en ventilación mecánica. Por tanto, se condujo un estudio observacional de obtención de ventanas ecocardiográficas en pacientes bajo anestesia general. Métodos: Se enrolaron 50 pacientes para cirugía abdominal. Luego del seteo del ventilador, en decúbito dorsal y con los campos quirúrgicos colocados se exploraron 4 ventanas: subcostal, apical, paraesternal y supraesternal. PEEP, volumen corriente, BMI, edad y sexo fueron registrados. Cada ventana fue evaluada con un score de accesibilidad. Resultados: La ventana subcostal no pudo ser obtenida en ningún paciente (campos quirúrgicos). De las 3 ventanas restantes sobre un score de 6 puntos posibles el promedio fue 5,14 . La ventana paraesternal obtuvo los scores más bajos $(p=0,0026)$. La causa más frecuente fue presencia del pulmón. No se encontró diferencia en el scrore de subgrupos (PEEP > 10 y BMI > 30) según el coeficiente de Pearson. Conclusión: La accesibilidad a las ventanas apical y supraesternal fueron muy buenas. La ventana paraesternal tuvo los menores puntajes pero no tuvo relación con el nivel de PEEP y el BMI. Limitaciones: el número de pacientes y condiciones del estudio no permite extrapolar los hallazgos a otros escenarios.

\section{Palabras clave:}

Accesibilidad, ecocardiografía transtorácica, anestesia general

\section{Introducción}

E xiste suficiente bibliografía que avala el uso de la ecocardiografía focalizada (FoCus) y el impacto que genera en la medicina crítica[1],[2],[3]. La Sociedad Americana de Ecocardiografía, conjuntamente con el Colegio Americano de Emergentógolos, declararon a FoCus como fundamental en el triagge de decisión en el paciente crítico[2]. En el departamento de urgencias y en la $\mathrm{UCl}$ existen programas educacionales para obtener competencia en FoCus en cuanto a la obtención de las ventanas ecocardiográficas y posteriormente su interpretación y contextualización con la clínica del paciente[5].

En anestesia hace más de 20 años que la ecocardiografía transesofágica (ETE) se utiliza en cirugía cardíaca, pero por diversas razones (morbilidad, formación y costos) no es una práctica que se haya generalizado hacia otros escenarios donde FoCus tendría especial utilidad[8].

La ecocardiografía transtorácica (ETT) presenta ventajas en relación al ETE en cuanto a su accesibilidad, morbilidad y repetibilidad. Existen numerosas publicaciones en distintos escenarios clínicos que reconocen la utilidad de la ETT en el contexto perioperatorio[12]. Sin embargo, en el paciente anestesiado durante el acto quirúrgico el acceso a las ventanas ecocardiográficas transtorácicas supone posiciones inmodificables, campos quirúrgicos y habitualmente en ventilación mecánica, todas situaciones que han sido señaladas como adversas para la realización de ETT[4].

Por lo tanto, decidimos conducir un estudio observacional en cuanto a la obtención de ventanas básicas ecocardiográficas en pacientes bajo anestesia general.

\section{Material y Métodos}

Se enrolaron 50 pacientes consecutivos para cirugía abdominal laparoscópica programadas entre junio y julio de 2017. Los estudios ecocardiográficos fueron realizados por operadores (autores) con formación avanzada (MMC y MC). Se incluyeron pacientes ASA I a III ente 18 y 80 años. Se excluyeron pacientes con cirugías cardíacas previas y alteraciones anatómicas visibles del tórax. Se realizó un flujograma describiendo la forma en que se reclutaron los pacientes que cumplieron con los criterios de inclusión, así como de las pérdidas de pacientes del estudio y los motivos de esas pérdidas.

Se realizó monitoreo para anestesia general normas FAAAR[10]. Luego de la inducción anestésica y 
la intubación orotraqueal se realizó el seteo del ventilador. Se utilizó ventilación protectiva con un volumen corriente (VT) entre 6-7 ml/k del peso teórico. Posteriormente, se realizó el Test del Aire[9]. Aquellos pacientes en que presentaban más de $30 \%$ de shunt se realizó una maniobra de reclutamiento alveolar para optimizar la oxigenación. Se estimó el punto de colapso y el nivel óptimo del nivel de presión al final de la espiración (PEEP) con la complacencia dinámica[13],[14]. Una vez seleccionada la ventilación mecánica (VM) en decúbito dorsal se procedece a la colocación de los campos quirúrgicos. Seguidamente, previo a la insuflación del abdomen, se valoró la exploración de las 4 ventanas ecográficas tradicionales apical, paraesternal, subxifoidea y supraesternal. Se utilizó un ecógrafo Phillips Sparq con una sonda phase array de 2,5 Mhz S4-2 (Philips Ultrasound 22100 Bothell-Everett Highway Bothell, WA 98021-8431 USA 2013). Se cronometró el tiempo en obtener cada una de las ventanas y se promedió el tiempo en hacerlo. Se valoró la posibilidad del acceso. Para ello se utilizó un score de accesibilidad de autoría propia pensada en la aplicación de ecocardiografía focalizada en el paciente crítico, priorizando la valoración cualitativa de imágenes en 2D en tiempo real que permitan tomar decisiones binarias y en donde el tiempo es importante. En caso de poder acceder a la ventana ecocardiográfica, se valoró con la siguiente puntuación: 0 punto (MALA) si no pudo obtenerse la ventana; 1 punto (BUENA) si se pudo valorar la ventana en un solo plano o dos planos sólo al final de la espiración o con pobre visualización del endocardio y 2 puntos (MUY BUENA) si se logró la vista completa de los dos planos en todo el ciclo ventilatorio y con buena visualización del endocardio. (Figura 1).

Se registró edad, sexo, nivel de PEEP, volumen tidal (VT) y BMI (body mass index).

Análisis estadístico: todas las variables se expresaron en promedio y 1 desvío estándar. La distribución normal fue testeada con la prueba de KolmogorovSmirnov. Se utilizó el test de Student para variables cuantitativas. Para establecer la correlación del score de accesibilidad en variables aleatorias (nivel de PEEP y BMI) se utilizó el coeficiente de Pearson. Para el análisis estadístico se utilizó el programa Epidat 4.2. Se estableció como nivel de significancia una $p<0,05$.

\section{Resultados}

Se expresan los datos demográficos en la Tabla 1. No hubo diferencia en el VT y nivel de PEEP en la población estudiada. De los 50 pacientes enrolados

\section{SCORE PUNTAJE}

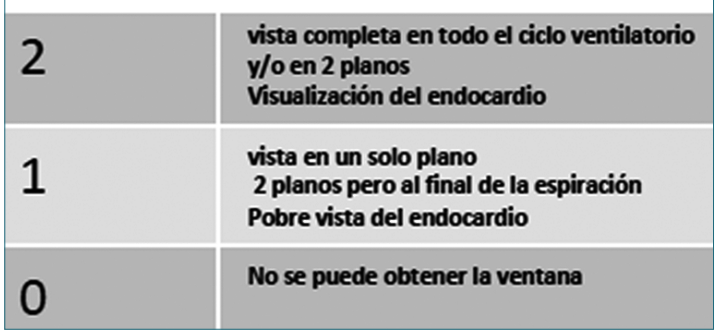

Figura 1.

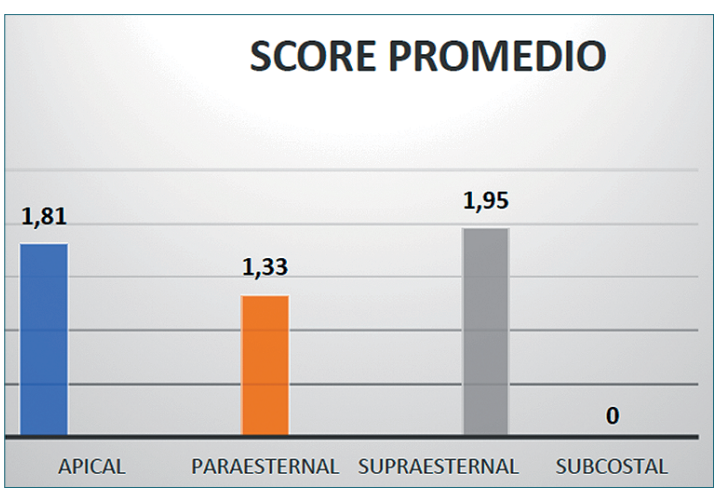

Figura 2. Comparación de score de accesibilidad por ventana ecocardiográfica. La accesibilidad a la ventana apical es superior a la paraesternal $(p=0,00026)$. La ventana supraesternal tiene el máximo score en los pacientes estudiados. $\mathrm{P}$ significativa $<0,05$.

se excluyeron 2 pacientes (prótesis mamarias) que alteraban la visualización de la ventana paraesternal. El acceso a la ventana subcostal no se pudo obtener en ningún paciente por la presencia de los campos quirúrgicos que se colocaron por encima del apéndice xifoides. Esta situación determinó la eliminación de la ventana subxifoidea del análisis. Evaluadas las 3 ventanas restantes sobre un score de 6 puntos posibles $(0$ a 2 cada una) el promedio obtenido fue de 5,14. Por lo tanto, la valoración general a las ventanas accesibles fue de buena a muy buena. El tiempo promedio en realizar los accesos fue $280 \pm 83$ segundos.

La ventana paraesternal obtuvo scores en promedio menor en relación a la apical ( $p<0,00026)$. La ventana supraesternal obtuvo el score de accesibilidad más alto. (Figura 2).

La causa más frecuente de imposibilidad de acceso a la ventanas fue la presencia del pulmón.

No se encontró relación entre BMI y la calidad de 


\begin{tabular}{lc}
\hline & Tabla 1. \\
\hline $\mathbf{n :} \mathbf{4 8}$ & Datos demográficos \\
Edad + sd & $51,6 \pm 17$ \\
Género \% & $\mathrm{F}: 28(48 \%)$ \\
& $\mathrm{M}: 26(42 \%)$ \\
$\mathrm{BMl}+\mathrm{sd}$ & $28 \pm 4,7$ \\
$\mathrm{VT}(\mathrm{m} / \mathrm{k})+\mathrm{sd}$ & $6,68 \pm 0,7$ \\
$\mathrm{PEEP}+\mathrm{sd}$ & $6,37 \pm 3,5$ \\
Score $(0-6)$ & 5,14 \\
\hline
\end{tabular}

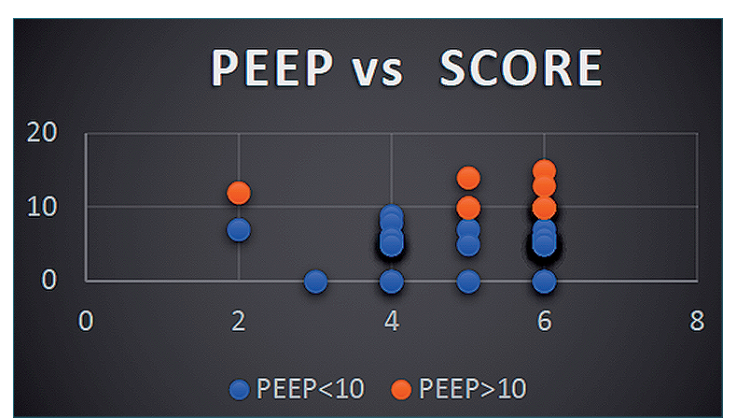

Figura 3. Relación entre el score de accesibilidad y el nivel de PEEP.

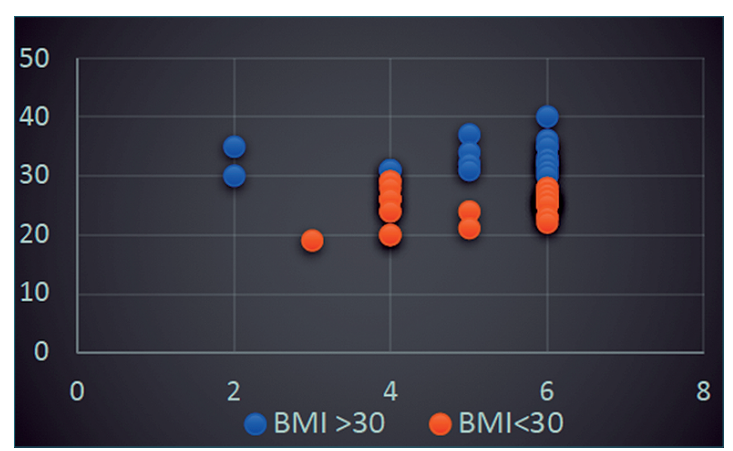

Figura 4. Comparación de score de accesibilidad en relación al BMI.

obtención de la ventana (Figura 3). En los niveles de PEEP y VT seteados tampoco se encontró correlación con score promedio obtenido por ventana (Figura 4).

\section{Discusión}

Varias publicaciones hacen referencia al impacto que genera calidad y número de ventanas obtenidas con ETT en pacientes en ventilación mecánica a la hora del diagnóstico y posterior guía de tratamiento en el paciente crítico[1]-[3]. En este estudio la ventana subxifoidea no tuvo posibilidad de acceso por el tipo de procedimiento. Sin embargo, Maitzel y col, publicaron una serie de pacientes en $\mathrm{UCl}$ donde demuestran que de las ventanas ecocardiográficas tradicionales, la suxifoidea es la de menor utilidad. Sólo 63\% presentó scores aceptables y eran de menor calidad en relación a $80 \%$ de acceso de la ventana apical. No hubo relación a la ventilación mecánica y al nivel de PEEP en este estudio[4].

La accesibilidad a ventanas en el estudio es en general muy buena. Sin embargo, la ventana paraesternal es la scores más bajos. La dificultad en el acceso se vio relacionada a la presencia del parénquima pulmonar. En el análisis de subgrupos no hay correlación entre la obtención y calidad de la ventana con el nivel de PEEP bajo ventilación protectiva. La hipótesis es que, en posición paraesternal en ventilación a presión positiva el pulmón se interpone frecuentemente entre el corazón y la pared torácica. No ocurre lo mismo con la ventana Apical. La explicación probable es la posición de la punta del corazón en contacto con la pared torácica por lo que la interferencia del pulmón en ventilación mecánica es menor. En la población estudiada no fue afectada esta situación con los niveles de PEEP y VT utilizados.

Varios artículos señalan a la obesidad como un limitante para la utilización de ETT[6],[7]. En este estudio no se observó correlación del BMI con los scores de calidad de obtención de las ventanas estudiadas.

La ventana supraesternal es la ventana con scores más elevados en este grupo de pacientes. Si bien no aporta información global del corazón permitiría va valoración del flujo midendo el IVT (integral de velocidad en el tiempo) de la aorta descendente. Esto es interesante para los anestesiólogos que a veces sólo tenemos acceso al cuello de los pacientes[11].

Este trabajo tiene limitaciones. El número de pacientes y la evaluación de las ventanas en una situación muy específica limita la posibilidad de extrapolar los resultados a otros escenarios clínicos.

\section{Conclusión}

La accesibilidad a las ventanas fue en general muy buena. Los campos quirúgicos impidieron el acceso a la ventana subcostal y la ventana para esternal tuvo menor acceso en relación a la presencia del parénquima pulmonar. No se encontró correlación entre esta situación con el nivel de PEEP y el BMI. 


\section{Referencias}

1. Via G, Hussain $A$, Wells $M$, Reardon R, ElBarbary M, Noble VE, et al. International Evidence-Based Recommendations for Focused Cardiac Ultrasound. Journal of the American Society of Echocardiography [Internet]. Elsevier BV; $2014 \mathrm{Jul} ; 27(7): 683 . e 1-683 . e 33$.

2. Labovitz AJ, Noble VE, Bierig M, Goldstein SA, Jones R, Kort $S$, et al. Focused cardiac ultrasound in the emergent setting: a consensus statement of the American Society of Echocardiography and American College of Emergency Physicians. J Am Soc Echocardiogr. 2010 Dec;23(12):1225-30.

3. FATE protocol. Eur J Anaesthesiol. 2004;21:700-7.

4. Maizel J, Salhi A, Tribouilloy C, Massy ZA, Choukroun G, Slama M. The subxiphoid view cannot replace the apical view for transthoracic echocardiographic assessment of hemodynamic status. Crit Care. 2013 Sep;17(5):R186.

5. Jozwiak M, Monnet $X$, Cinotti $R$,
Bontemps F, Reignier J, Belliard G. Prospective assessment of a score for assessing basic criticalcare transthoracic echocardiography skills in ventilated critically ill patients. Ann Intensive Care. 2014 Apr;4(1):12.

6. Value and Limitations of Echocardiography in the Assessment of Obese Patients J of CV ultrasound and Allied Techniques May1986.

7. Benoy N, et al. Stress echocardiography in patiens whith morbid obesity. Echo Res Pract. 2016 Jun;3(2).

8. Sasha K. Shillcutt, MD, FASE* and Julian S. Bick, MD†A. Comparison of Basic Transthoracic and Transesophageal Echocardiography Views in the Perioperative Setting AnesthesiaAnalgesia. 2013 Jun;116(6):1231-5.

9. Tusman G, Groisman I, Fiolo FE, Scandurra A, Arca JM, Krumrick $G$, et al. Noninvasive monitoring of lung recruitment maneuvers in morbidly obese patients: the role of pulse oximetry and volumetric capnography. Anesth
Analg. 2014 Jan;118(1):137-44.

10. www.anestesia.org.ar/normas

11. Royse CF, Royse AG, Soeding PF, Mathieson EM. Descending aortic pulsed wave Doppler can predict changes in cardiac output during off-pump coronary artery bypass surgery. Ann Thorac Cardiovasc Surg. 2003 Oct;9(5):314-8.

12. Royse CF, Canty DJ, Faris J, Haji DL, Veltman M, Royse A. Core review: physician-performed ultrasound: the time has come for routine use in acute care medicine. Anesth Analg. 2012 Nov;115(5):1007-28.

13. Rama-Maceiras P. Atelectasias perioperatorias y maniobras de reclutamiento alveolar. Arch Bronconeumol. 2010 Jun;46(6):317-24.

14. Suárez-Sipmann F, Böhm SH, Tusman G, Pesch T, Thamm O, Reissmann $\mathrm{H}$, et al. Use of dynamic compliance for open lung positive end-expiratory pressure titration in an experimental study. Crit Care Med. 2007 Jan;35(1):214-21. 